\title{
Topological Invariance of Biological Development
}

\author{
Eugene Presnov $\cdot$ Valeria Isaeva $\cdot$ Nikolay Kasyanov
}

Received: 7 February 2013/Accepted: 29 April 2013/Published online: 12 May 2013

(C) The Author(s) 2013. This article is published with open access at Springerlink.com

\begin{abstract}
A topological inevitability of early developmental events through the use of classical topological concepts is discussed. Topological dynamics of forms and maps in embryo development are presented. Forms of a developing organism such as cell sets and closed surfaces are topological objects. Maps (or mathematical functions) are additional topological constructions in these objects and include polarization, singularities and curvature. Topological visualization allows us to analyze relationships that link local morphogenetic processes and integral developmental structures and also to find stable spatio-temporal topological characteristics that are invariant for a taxonomic group. The application of topological principles reveals a topological imperative: certain topological rules define and direct embryogenesis. A topological stability of embryonic morphogenesis is proposed and a topological scenario of developmental and evolutionary transformations is presented.
\end{abstract}

Keywords Discrete curvature - Morphogenetic field · Packing · Polarity · Singularity · Topological information - Topological polyhedron · Topological surgery · Voronoi structure

\footnotetext{
E. Presnov $(\bowtie)$

The Volcani Center, 85280 Beit Dagan, Israel

e-mail: epresnov@agri.gov.il

V. Isaeva

Institute of Ecology and Evolution, Moscow 119071, Russia

e-mail: vv_isaeva@mail.ru

V. Isaeva

Institute of Marine Biology, Vladivostok 690041, Russia

N. Kasyanov

Institute of Theory and History of Architecture and Town Planning, Moscow 105264, Russia e-mail: kas_nv@mail.ru
} 


\section{Introduction: Local and Global Order}

The term topology was first introduced by Johann Listing (1848), who gave the following definition of topology: "By topology we mean the doctrine of the modal features of objects, or of the laws of connection, of relative position and of succession of points, lines, surfaces, bodies and their parts, or aggregates in space, always without regard to matters of measure or quantity". It is well known that topology deals with the most general properties of spaces as mathematical objects (Bourbaki 1948) which concern, first and foremost, local-to-global transitions. The classical description of natural forms does not contain topological elements (Rossi 2006). Obviously, biological processes during embryonic development have stable spatial features and stable temporal dynamics (global order). It is also clear that living organisms inhabit and develop in the real physical space and are organized according to the global topology of this space (Gromov 2011). Nicolas Rashevsky (1958) was the first to apply a topological approach to biology which he named biological topology or shortly-biotopology.

René Thom (1969, 1996 ) put forward a topological description of embryogenesis and argued that quality cannot be reduced to quantity, as the discrete character of biological morphogenesis entails qualitative discontinuities: "there have indeed been qualitative innovations in evolution". The topological approach to the description of biological morphogenesis has become a more common practice (Presnov 1982; Maresin and Presnov 1985; Allaerts 1999; Jockusch and Dress 2003; Baas and Seeman 2012; Honda 2012; Isaeva et al. 2012; Shimizu 2012; Morozova and Shubin 2013). We follow below the topological perspective "Life without metric" by Mikhail Gromov (2010) using elementary topology to describe structural dynamics of biological forms and morphogenetic processes in ontogeny and evolution.

We consider well-known mathematical axiomatics for an extended topological description of biological development. As early as the 18th century, Leonhard Euler had proposed that all geometric figures consist of three fundamental elements: lines (or trajectories), vertexes (or crossings) and areas (or openings). A polyhedron is a geometric figure, which is the 3D version of the plane polygon. It is a finite connected set of polygons joined together, bounding a volume in such a way that each side of every polygon is tangent to (connected to) a side of exactly one other polygon.

Now we introduce other fundamental definitions that will be useful below.

Topological equivalence $=$ homeomorphism. A map $f: X \rightarrow Y$ between two topological spaces is called a homeomorphism if it has the following properties: (1) $f$ is one-to-one and onto (bijection), (2) $f$ is continuous, (3) the inverse map $f^{-1}$ is continuous ( $f$ is an open map).

Homotopy $=$ continuous deformation . A homotopy between two continuous maps $f$ and $g$ from a topological space $X$ to a topological space $Y$ is defined to be a continuous map $H: X \times[0,1] \rightarrow Y$ from the product of the space $X$ with the unit interval $[0,1]$ to $Y$ such that, if $x \in X$ then $H(x, 0)=f(x)$ and $H(x, 1)=g(x)$.

Given two spaces $X$ and $Y$, we say they are homotopy equivalent (the same homotopy type) if we can find continuous maps $f: X \rightarrow Y$ and $g: Y \rightarrow X$ such that 
$g \circ f$ is homotopic to the identity map $X \rightarrow X$ and $f \circ g$ is homotopic to the identity map $Y \rightarrow Y$. Shrinking, stretching and bending are possible (angles and distances are not preserved). Cutting and pasting are impossible. So we can note that, for instance, the number of holes in a 2D surface is a homotopy invariant. Clearly, every homeomorphism is a homotopy equivalence, but the converse is not true: for example, a solid ball is not homeomorphic to a single point, although the same ball and the point are homotopy equivalent. We will also use the term simply connected manifolds (any closed curve drawn within the manifold can be shrunk to a point, where the shrinking stays within the manifold).

Let $X$ be a topological space homotopy equivalent to some cell complex ( $C W-$ complex). Then a homotopy dimension of $X$ is the minimal number $k$ such that $X$ is homotopy equivalent to a cell complex of dimension $k$.

Initial local-global keys in animal development are oogenesis and fertilization (Presnov and Isaeva 1990; Isaeva et al. 2012) (Sects. 2 and 3). The spatial arrangement of blastomeres of an early embryo is a stable feature of taxonomic value, which is evolutionarily and genetically determined (Sect. 5) During early development, form creation involves varying arrays of polyhedrons. Since the present work focuses on the morphological structuring of early pattern formation, we shall review some of the basic assumptions. Early cell communities sequentially replacing one another are structurally stable (global order) throughout intervals between special local modifications_ruptures and gluings (local order). Topology of cell connections inside an embryo will be described (Sect. 6), since interactions between neighboring cells play a crucial role during embryogenesis (Isaeva et al. 2006; Chuong et al. 2006).

During development, the topological constituent of embryo architecture decreases, and detailed elaboration of geometrical form takes place. A blastula has an inner cavity, blastocoel, surrounded with primary germ monolayer, so it is an epithelial sphere, formed with embryonic cells joined by specialized contact junctions (Sect. 7). Since a sphere (topologically) has an inside and outside, blastula formation results in cell polarization (Sect. 2). The process of making a set of oriented epithelial cell layers (and transferring from 0 to 2 homotopy dimension) is called gastrulation (Sect. 5). This process represents the first morphogenetic movement in the developing embryo: shaping the archenteron, a primary gut (Sects. 8 and 9).

Various scalar and vector fields at all levels of biological organization are revealed as heterogeneous distribution of structural components, functional activities, and biochemical gradients. Trans-cellular and -epithelial ion currents generate electric fields as signals and effectors of cell polarity. It is known that the distribution of certain sets of mRNAs in the egg ooplasm determines the spatial pattern of the future embryo (Nuccitelli 1984; Nüsslein-Volhard 1991; Gilbert 2010); spatial anisotropy in distribution of gene products creates a morphogenetic field (scalar concentration field and resulting vector polarity field) specifying the polarity of future organism (Sect. 2) Spatial images of the biological fields are analyzable by topological zoom; singularities inevitably emerge and transform during the period of embryo development. It is the topological approach that is capable of providing a correct and adequate description of developmental dynamic 
architecture. A vector field is a section of a corresponding tangent bundle (see Dubrovin et al. 1990, 1992, 1995). So we need to find a suitable section of a tangent bundle to fix a real topological criterion of early development. Animal development is serial topological rearrangements of 2D closed surfaces (Sect. 10) This conceptual accent allows us to indicate topological simulations of onto- and phylogenesis (Sect. 11)

\section{Polarization: Singular Point}

The boundary of the ball We shall begin ab ovo. In most metazoans, the animalvegetal/anterior-posterior axis of an egg and a future embryo is established during oogenesis and usually depends on the position of an oocyte in relation to its cellular and non-cellular surrounding. A polarized cellular architecture is fundamental to the establishment of anterior-posterior polarity. Both the actin and microtubule cytoskeletal elements work together to convert an initial asymmetry into a global cell polarity (Li and Bowerman 2010; Mullins 2010). Egg cytoskeleton functions as a global morphogenetic determinant, which directs and maintains the anisotropic distribution of ooplasmic molecular information, which determines the axial polarity of an egg and a future organism (Isaeva et al. 2008). It has been experimentally shown that the main carrier of molecular morphogenetic information in an oocyte/unfertilized egg is its cortical layer, a thin peripheral layer of ooplasm.

There is a vector morphogenetic field on the egg surface (2-sphere). The cortical egg field is visualized, in particular, by polarized distribution of membrane and cytoskeletal components and by the electric field. For example, the axial polarity of oocytes in some animals is manifested in transcellular ion flows, which generate the extracellular electric field (Fig. 1a). The oocyte/egg cortical field is the morphogenetic one, since it determines the polar axis (or axes) of future organism; the polarized cortical layer is the carrier and indicator of the vector field on the egg surface (Presnov et al. 1988; Presnov and Isaeva 1990).

Topological singularity arises as a consequence of a symmetry breaking process, when a system goes through the phase transition from the spherical symmetry to a broken symmetry. Then, according to the well-known Poincaré-Hopf theorem (Milnor 1965):
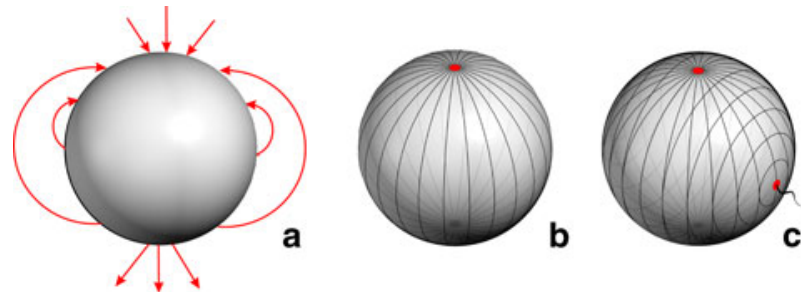

Fig. 1 Egg polarization: a the electric field of the frog oocyte (after Nuccitelli, 1984); b animal pole singularity; c second singularity at site of sperm penetration 
For any continuous tangent vector field given on a 2-sphere, a singular point of the field exists.

The occurrence of a vector field singularity (or singularities) on the sphere is/are inevitable. This singularity breaks the topological symmetry of the egg, polarizing the egg sphere. This theorem is also called Hairy ball theorem: "you can not comb a hairy ball flat without creating a cowlick". It means a 2 -sphere has none trivial tangent bundle and therefore a 2-sphere is not parallelizable.

This general remark about vector fields is possible given concrete expression. We suggest a topological explanation for any polarization on a single cell like an egg. Patently, the boundary surface of this cell is homeomorphic to a standard 2-sphere (Sect. 5). First of all, any scalar function (for example, the concentration of any substance) on the sphere produces a gradient of this function. This gradient gives a polarization on the sphere. We must talk about a vector field on the spherical surface of this cell. In this case we use a decomposition of vector field into gradient component and another nongradient one. For this purpose, there is the theorem (Presnov 2008), which is most interesting here in cases (3D ball $B^{3} \subset \mathbb{R}^{3}$ and 2-sphere $S^{2}=\partial B^{3}$ ):

For any smooth vector field $v(x)$, there exists a unique global decomposition:

$$
v=\operatorname{grad} \varphi+u_{\odot} .
$$

The potential $\varphi(x)$ of the gradient vector field has direct computation. The additional field $u_{\odot}(x)$ is the concentric vector field preserving the foliation of spheres into ball $B^{3}$ (or circles on $S^{2}$ ). In other words, we have the dissipative-free vector field $u_{\odot}(x)$ with concentric structure. See also another Helmholtz-Hodge decomposition of vector fields (Fuselier and Wright 2009).

\section{Sperm Penetration: Another Singular Point}

In most metazoans, the first singular point(s) (egg poles) appears during oogenesis (Fig. 1b). In some animals, at least in chordates, in addition to preexisting animal and vegetal egg poles, a new singularity emerges at the site of sperm penetration. This new singular point determines the dorsal-ventral axis of the egg and future organism (Fig. 1c). So, in chordates, the second symmetry breakdown takes place following gamete contact and fusion. For example, in an oocyte of the frog Xenopus, the anisotropic distribution of specific maternal mRNAs and proteins along an animal-vegetal axis underlies the anterior-posterior patterning of the future embryo; a cortical rotation after insemination produces an additional symmetry breaking that creates the dorsal-ventral axis (Newport and Kirschner 1982).

Different organisms use various biological mechanisms and time schedules to establish anterior-posterior and dorsal-ventral polarity. In Drosophila, both polar axes are determined during oogenesis, while in most animals only animal-vegetal axis is determined before egg fertilization. In the nematode Caenorhabditis elegans, anterior-posterior polarity is specificated by sperm entry. During sperm penetration 
in echinoderm and mouse eggs, the fertilization cone arises by polymerization of actin filaments. As it was shown in frogs and sea urchins, the plasma membrane of a spermatozoon is inserted into the egg plasma membrane, and the sperm surface remains localized at the site of the penetration; this spot is visible during early cleavage. Following gamete fusion in mice, polar body emerges; in egg/polar body contact zone, the accumulation of actin and spectrin was observed (Shapiro et al. 1981; Johnson and Maro 1985; Kirschner et al. 1985; Reima and Lehtonen 1985; Kyozuka 1993; Goldstein and Hird 1996).

So the local order of sperm penetration is connected to the integral order of the egg, zygote and developing organism. The inevitable breaking of the preexisting symmetry pattern is programmed both genetically and epigenetically and plays a critical role in embryogenesis.

\section{Ooplasmic Segregation: Fixed Point}

The rearrangement of the egg cortex organization following sperm penetration involves ooplasmic segregation. Ooplasmic segregation was studied in eggs of ascidians and the annelid Tubifex as the flow of cortical actin networks triggered by the sperm penetration and generating the spatial-temporal vector field of the cortical displacement. In ascidians, the yellow cortical pigment moves to the vegetal pole after sperm penetration and at the following stage forms a yellow crescent-like region somewhat lower the egg equator. In amphibia, the cortex of inseminated egg is restructured with displacement of the pigment in animal area and rotates in relation to the inner ooplasm with formation of so-called gray crescent (Sawada and Osanai 1985; Sawada 1988; Shimizu 1988, 1989; Kirscher and Gerhart 2005).

So the symmetry breaking process in chordates involves the flow of the cortical actin network. The rearrangement of ooplasm and emergence of both ascidian yellow crescent and the amphibian gray crescent is involved in the establishment of the dorsal-ventral axis of future animal. Thus, the local order of ooplasmic segregation is connected to the integral order of the developing organism. Ooplasmic segregation results in a spatial-temporal vector field of the displacement of cortical components. During cortical reorganization, subcellular, mainly cortical components of an egg are moving with respect to a fixed, stationary point in the course of continuous deformation. This is the fixed point of the ooplasmic flow. The fertilized egg, zygote is topologically equivalent to the ball. During cytoplasmic segregation as well as in similar processes of integral cell polarization such as chemotaxis, capping or attachment to a substrate, we may assume a mapping a ball into itself. According to Brouwer's theorem:

Any continuous map of the closed ball into itself has a fixed point.

This fixed point is an egg pole or additional singular point, which serves as a source or sink during ooplasmic segregation. This point breaks the spherical symmetry of the egg. Thus, the order of ooplasmic localization is connected to integral order of the egg.

Note that, in normal development, there is a single fixed point during the period of ooplasmic rearrangement; at the same time some experimental influences can 
cause the appearance of additional singularity. For example, in amphibian eggs, abnormal gravitation vector imposed immediately after insemination can produce two-headed embryos. Fundamental symmetry transformations at the cell level provide the most important morphogenetic information determining axial coordinates of future animal. Thus, ooplasm possesses irreversible epigenetic memory of an imprinting kind.

\section{Early Cleavage: Cell Complex}

A cleaving zygote is a set of connected blastomeres defining the embryo geometry. Each blastomere has a spherical surface. This closed spherical surface of a blastomere fragments into cell contact domains. Let us mentally expand all these contact areas; we blow up each this face in such a manner that it will border with other domains on the surface of a given blastomere. These regions can be considered as curved faces of generalized polyhedron. As a consequence each blastomere is not a geometrical polyhedron but a spherical one. The result of this technical procedure is very similar to the Voronoi structure. These polyhedra are topological ones: a space homeomorphic to a geometrical polyhedron is called a topological polyhedron (t-polyhedron). We are obligated to talk about cell complex as a topological approximation of biological tissues (Hoffman 1973).

Here we examine the building of an early embryo during an egg cleavage (cell division). Each blastomere is modeled as a topological cell, and a whole embryoas a cell packing. The egg cleavage results in the emergence of a net of cellular contacts on the surface of an embryo, a discrete morphogenetic field (Sect. 6).

Zygote cleavage is cell mitotic reproduction and results in 2, 4, 8 and so on blastomeres. Dense packing of early 8-16 blastomeres is the morula (so named because it is similar to mulberry, a berry of Morus). The morula is a cellular close packing, which is topological equivalent to a ball (3D object). But any ball is homotopy equivalent to the point. The dimension of any point is zero. Morphogenetic modifications of this spatial object without topological motivation looks like a fresco on a wall. In mammalian embryogenesis, 8-cell morula undergoes a process called compactization: individual cells are joined by tight junctions and form a ball.

To summarize, we asked which topological change of an early cleaving embryo inevitably happens with the increasing number of its cells. To answer this question, we used polyhedral tessellations. However, this model is insufficient to describe the developmental stages beyond the morula.

\section{Axis Formation: Discrete Gradient}

The problem of axis formation has a long story and it raises numerous questions (Allaerts 1991). We want to discuss the natural topological structure of the morula that can determinate a certain morphogenetic field on the boundary of the morula.

Alexander Gurwitsch (1922) was the first to introduce the concept of "anisotropic cellular field" into biology. The presence of such a field is related to 
the vector character of the subcellular organization with the oriented mutual positioning of molecules and ordering of molecular "constellations". He assumed that the field has a radial structure with a center localized in the cell nucleus; the cellular field is continuous and divides during cellular divisions. The fields of neighbouring interacting cells unite into one "actual" field which is the vector sum of its cellular field components. The theory of Gurwitsch, specified and illustrated geometrically as a vector system, was accepted by contemporaries and is considered by numerous biologists nowadays to be a scholastic, purely abstract one and as a formalism which has nothing to do with real life. But Gurwitsch's concept is strengthened by the fact that the morphological polarization of cells provides a set of vectors in embryonic tissue, which can be considered as a predicting factor of the future tissue formation possessing a status of cause-effect mechanism (Beloussov 1998). It can have a support from the following point.

Specially, here we want to think about another conception for simulation of those fields. So we modeled any morula as a finite cell complex. On the combinatorial surface $\mathcal{K}$ of this 3D cell complex, a discrete vector field could exist (Forman 1998). For our application, let us assume that $\mathcal{K}$ is a combinatorial surface and $K$-the set of cells. The cell $\sigma$ is a face of $\tau$, denoted by $\sigma<\tau$, if $\sigma$ is contained in the boundary of $\tau$. Facets are the faces of codimension 1.

A discrete vector field $V$ on the combinatorial surface $\mathcal{K}$ is a set of pairs of cells $(\sigma, \tau) \in K \times K$, with $\sigma$ a facet of $\tau$, such that each cell of $K$ belongs to at most one pair of $V$ (local order).

A $V$-path $\Gamma$ of a discrete vector field $V$ from a cell $\sigma_{0}$ to a cell $\sigma_{r}$ is a sequence $\sigma_{0} \tau_{0} \sigma_{1} \tau_{1} \ldots \tau_{r-1} \sigma_{r}$ of cells such that for every $0 \leq i \leq r-1$ :

$$
\begin{aligned}
\sigma_{i} \text { is a facet of } \tau_{i} \text { and } & \left(\sigma_{i}, \tau_{i}\right) \in V, \\
\sigma_{i+1} \text { is a facet of } \tau_{i} \text { and } & \left(\sigma_{i+1}, \tau_{i}\right) \notin V .
\end{aligned}
$$

A $V$-path is a nontrivial closed path if $\sigma_{0}=\sigma_{r}$ and $r>0$.

A discrete gradient vector field is a discrete vector field $V$ that does not admit any nontrivial closed $V$-path (global order). A discrete gradient vector field might be understood as the gradient of some function in the following sense.

A discrete Morse function is a function $f: K \rightarrow \mathbb{R}^{1}$ on the cells of a regular combinatorial surface $\mathcal{K}$ if there is a gradient vector field $V$ such that for all pairs of cells $(\sigma, \tau)$ is true

$$
\begin{cases}f(\sigma)<f(\tau) & \text { if }(\sigma, \tau) \notin V, \\ f(\sigma) \geq f(\tau) & \text { if }(\sigma, \tau) \in V,\end{cases}
$$

here $\sigma$ is a facet of $\tau$.

Accordingly, if the combinatorial spherical surface of the early embryo (morula) has a discrete Morse function, then it means that polarization of this surface follows in accordance with a corresponding discrete gradient vector field. Otherwise, every discrete vector field on the combinatorial spherical surface has a closed $V$-path. This closed $V$-path divides the closed spherical surface in two different parts and therefore it can be concluded that polarization takes place in this case too. 


\section{Blastulation: Orientable Surface}

After cleavage, the epithelization of the primary germ layer and the progressive switch from a maternal to a zygotic control of embryo patterning- "mid-blastula transition"- takes place at different stages according to a species. The mid-blastula transition was studied mainly in echinoderms and vertebrates, first and foremost, in amphibia. In echinoderms, the switching of cell interactions occurs when the embryo consists of 128-256 cells. In amphibians, this switch is also carried out at the mid-blastula consisting of about 4,000 cells (Newport and Kirschner 1982; Kirschner and Gerhart 2005; Gilbert 2010). In mammalian embryos, the first epithelized cell layer (trophectoderm) is the extraembryonic one, which is required for implantation and development of the placenta. The preimplantation development involves six cleavage divisions before blastocyst formation from the morula. Blastocyst consists of approximately 60 cells, with an inner cell mass and specialized outer trophectoderm cells. The embryo develops from the inner cell mass (Geneviere et al. 2009). A mammalian embryo by the cavitation process becomes the blastocyst.

Epithelial cell layers of a metazoan organism are characterized by structural and functional connectivity, closeness of intact surfaces and outer-inner anisotropy of the epithelial layer. To translate anatomical descriptions of metazoan morphology into topological analogies, the external shape of an organism is modeled by smooth closed surfaces. The connectivity of an epithelial layer during epithelial morphogenesis is ensured by the system of specialized intercellular contacts that integrate cells into a morphological and functional entity (Kolega 1986; Zallen 2007; Gibson and Gibson 2009). The appearance of the epithelial tissue was one of the most significant innovations in the early metazoan evolution; the steps involved were specializations of intercellular contact complexes and extracellular matrices.

In early cleavage and at the morula stage, blastomeres have inner contacts. This set of contacts is inhomogeneous. Blastomeres do not maintain a large number of contacts. Large cells are not stable and divide into smaller cells. An increasing number of cells during early cleavage leads also to the increasing number of contacts per cell and subsequent differentiation.

In an attempt to understand how inside-outside positions give rise to the cell polarity establishment, a possibility which we can name a topological scanner for apical-basal polarization has been put forward.

The inside-outside and cell polarity phenomena are complementary, because both rely on differences along the radial axis breaking the symmetry of the early embryo. As a result of epithelization, the external surface of an embryo becomes smoother and more spherical; blastocoel, the inner cavity filled with a liquid, appears. The epithelized cell sheet has potential motility (it is not a $3 \mathrm{D}$ close packing) that gives a possibility of further morphogenetic movements in early development.

The first topological rearrangement of embryo morphology is the appearance of the separated two-side closed sheet of epithelial cells. Indeed the birth of the cavity is a complication of topology. Cavity creation modifies the topology of cell ensemble and this $3 \mathrm{D}$ cell complex becomes topologically equivalent to the sphere. 
In most animals, cavity of blastocoel emerges during cleavage and enlarges in consequence of germ layer epithelization. In mammals, the cavity of the blastocyst is not a blastocoel. Mammalian morula is transformed to the blastocyst through delamination. In mammalian embryogenesis, cell fate is specified at the 8-cell stage by the establishment of cell polarity (Yamanaka et al. 2006; Johnson 2009) along the radius of early morula. Subsequent cell divisions lead to symmetric or asymmetric distribution of polarity information, based on the angle of cell division. Division parallel to the radial axis generates two identical, polarized cells that inherit the entire distribution of polarity information. In contrast, asymmetric division "perpendicular" to the inside-outside axis creates two different cells: outside one, and one apolar cell that stays inside. Both cell types would then differ in their developmental potential.

Mechanism of cavity formation Thus, the cavity formation is important in animal development, and this is a good example of a simple form that develops into a complex form. Blastula is a 3D cell complex of close packing polarized cells, and its cells have coherent positions from one to another. This coherence creates a virtual discrete field formed by cell structure vectors. Cells have "almost parallel" structure vectors, which slowly change from one cell to another. We can mark a smooth vector field in the whole volume of morula or blastula, and on the boundary of an embryo this field is directed inside.

The next step of the simulation is: this vector field generates a continuous flow (map). And this map, by Brouwer's theorem (Sect. 4) has a fixed point (or zero of corresponding vector field). Eventually, we can distinguish an inner cell with zero vector of polarization. So cells produce a cavity. It is a topological event-a cavity building. It means the appearance of a closed cell layer.

Planarity of graph of blastomeres Cell contacts maintain a closed cell layer and entire embryo as a whole. A frontier epithelial area has a free surface (local order) bordering an environment. The set of epithelial cells forms a closed two-side spherical envelope (global order) of an embryo. Each boundary cell having its "free" face is polarized (Presnov et al. 2010).

The problem is: we chose for initial set either some cells or even no cells. In the case of a small number of blastomeres of an early embryo, we cannot pick out an outer cellular sheet. A critical blastomere number (which is a specific for given species) forms a new topological landscape-an external spherical layer, which contains all cells of an embryo. These external cells are polarized. This local order leads to the emanation of the global order-an external spherical layer with a planar graph of cell contacts. However, it can be picked out from a bond graph of a spherical cover. Probably a minimal number of blastomeres for detection of closed spherical cover is equal 4 . In this case, blastomeres place at the edges of a tetrahedron (planar graph $K_{4}$ ). For a nonplanar blastomere graph with the minimal number of nodes 5 (Kuratowski graph $-K_{5}$ ), by picking out a planar margin subgraph it is eliminated an edge inside as physically unreal one (Fig. 2). The closed spherical coat from close packing cells has a boundary function of separation from an environment and therefore has a topological reason for a cavity appearance. 
Fig. 2 Planar graph $K_{4}$ and minimal nonplanar graph $K_{5}$

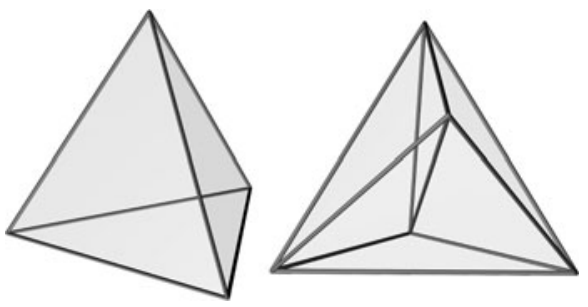

\section{Gastrulation: Discrete Curvature}

In mathematics, our attention focuses on geometric properties of the boundaries of domains and on the influence of those geometric properties on the complex analysis of the domain. Using a model of close spatial cell packing and appearance on the blastula surface a cell tessellation, we introduce the natural assumption of curvature (local order), which is initiated by this tessellation. This leads to the appearance of cells with negative curvature. We possess a topological prediction and argument of invagination, a topological cause of an immersion inside the spherical surface. Returning to polyhedral surfaces it is useful to fix a notion of curvature, independent of all geometrical information. For each graph on the sphere, a face is characterized by the number of neighboring faces, and this implies that curvature can be set up in case of the polyhedral surface with canonical Euclidean metrics for the 3D space. We give here a general definition of polyhedral objects; this leads to another interpretation of the curvature of a polyhedral surface. A polyhedral 3D object $P^{3}$ means a cell complex which is homeomorphic to a $3 \mathrm{D}$ object, which is regular and satisfies the intersection condition. That is, each cell is embedded in $P^{3}$ with no identifications on its boundary, and the intersection of any two cells (of any dimension) is a single cell (if nonempty). Because $P^{3}$ is topologically a manifold, each face is contained in exactly two cells (face to face).

The idea how to define a discrete (combinatorial) curvature was presented in our previous works (Presnov and Isaeva 1990; Presnov and Isaeva 1991) in a simple topological computation: on the surface of fertilized and cleaving egg $\left(S^{2}\right)$, the discrete morphogenetic field emerges as a pattern of blastomere contacts. For every face $f$, a value $k(f)$ of the discrete curvature of this face

$$
k(f)=1-\frac{|f|}{6}
$$

can be defined. This discrete curvature of a face on the $3 \mathrm{D}$ cell obeys the discrete Gauss-Bonnet theorem. The classical Gauss-Bonnet theorem for the sphere says

$$
\frac{1}{2 \pi} \int_{S^{2}} k d \sigma=2 .
$$

Here $k$ is the Gaussian curvature. As a consequence of the Euler theorem (Hirzebruch and Kreck 2009) for structurally stable graphs on the sphere, $\sum_{f} k(f)=2$. Therefore, $k(f)$ is the discrete analogue of continuous value $k$. 
Remark The same approach was proposed elsewhere. A corner of a tessellating graph is a pair $(v, f) \in V \times F$ such that $v \in \partial f$. The set of all corners of $G$ is denoted by $C:=C(G)$. Let $G$ be a plane tessellation. Then the function $C \rightarrow \mathbb{R}^{1}$ defined by

$$
k(v, f):=\frac{1}{|v|}+\frac{1}{|f|}-\frac{1}{2}
$$

is called the discrete curvature on the graph $G$ (Knill 2012). The definition of discrete curvature is relevant for planar graphs only.

The egg cleavage results in a pattern of cell contacts on the surface of the embryo as a discrete morphogenetic field. The epithelization of the primary germ layer takes place at different stages according to the species. In many animals, during midblastula transition, a loose packing of blastomeres is replaced by densely packed cells, which form on the embryo surface a network of polygons by increasing the length of specialized contact zones. In the period corresponding to mid-blastula transition, dissociated blastomeres of a starfish or sea urchin form the epithelized blastula by bending and closure of a fragment of the cell monolayer, by a process, called blastulation (Dan-Sohkawa and Fujisawa 1980; Kadokawa et al. 1986; Presnov et al. 2010).

We observed a similar blastulation process in experiments with dissociated blastomeres (embryos of the sea urchin Strongylocentrotus nudus). It was shown earlier that sea urchin embryos formed from reaggregated blastomeres are possible to develop into larvae and transform after metamorphosis into fertile sea urchins (Giudice 1962; Hinegardner 1975). The transition of the cytoskeleton/cytomatrix into integrated "histoskeleton" by means of intercellular integration through cellcell contacts gives the possibility of coordinated epithelial movement. Epithelial movements are responsible for many morphogenetic transformation, including the folding, delamination, cavitation (Kolega 1986). Some topological inhomogeneities presumably can determine the localization and direction (inside or outside) of the epithelial morphogenetic movements.

There are only five homogeneous $(k=$ const $)$ discrete fields on the sphere, which correspond to five regular polyhedra, and only three of them are structurally stable $\left(\mathbf{3}_{\mathbf{4}}, \mathbf{4}_{\mathbf{6}}\right.$ and $\left.\mathbf{5}_{\mathbf{1 2}}\right)$. During synchronous cleavage divisions, only the first four blastomeres admit a homogeneous field on the embryo surface. Later the field of cellular contacts on the embryo surface inevitably becomes topologically inhomogeneous. Consequently, the symmetry of the discrete morphogenetic field breaks. The occurrence of inhomogeneity of this field on a sphere, in accordance with Euler's theorem used for polyhedra, is a consequence of the topology of our 3D physical space. So, in conformity with biological terminology, positional information for discrete morphogenetic fields is found out by theorems of Gauss and Euler as a relationship between its local order and the integral order. Probably it is preferable here to use a more specific term-topological information. As soon as the sum of adjacent faces becomes larger than 6, the local face curvature becomes negative, which leads to invagination and, consequently, to gastrulation. When the number of adjacent faces is smaller than 6, the face's curvature is positive (Presnov et al. 2010). 
An active epithelial morphogenesis was revealed in experiments in vitro: starfish zygotes without the fertilization envelope after several cleavage divisions formed a cell monolayer, finally closing into a hollow sphere resembling a normal blastula (Dan-Sohkawa and Fujisawa 1980). It was shown that during this blastulation process, the presence of the cells with negative curvature correlates with the incurvation of the cell sheet (Presnov et al. 2010).

It was found also that in some animals, the localization of invagination or other type of morphogenetic cell movements during normal embryogenesis coincides with the position of a cell having the highest value of the cell field as negative curvature (Presnov and Isaeva 1991; Isaeva et al. 2012). For example, Fig. 3 demonstrates discrete singularities of the cell contact field on the surface of animal and vegetal semi-spheres of a crustacean embryo: the localization of the cell with negative curvature coincides with the site of cell ingression during the following gastrulation, while cells of positive curvature are localized on the animal surface of the embryo.

Epithelial cell layers in metazoan organisms are characterized by outer-inner anisotropy. Two sides of a cell sheet may also differ in the number of cells and cell contacts able to determine the vector of an epitheliogenesis: incurvation (invagination) or excurvation (evagination) of the cell layer (Presnov et al. 2010).

Obviously, gastrulation is not directly related to the inhomogeneity of the blastomere pattern following cleavage. Rather, the place on the egg surface having the highest value of the cell field and the localization of morphogenetic cell movement in gastrulation coincide because they both are determined by preexisting pattern of embryo polarity (Presnov and Isaeva 1991). Conversely, cells with the lowest number of contacts become centers of evagination and proliferation. A similar concept was previously put forward to explain cell proliferation in intestinal macrovilli (Pyshnov 1980; Klein 2002; Rumpler et al. 2008). Thus, initiation of gastrulation may be related to the inevitable emergence of local curvature in the discrete morphogenetic field on an embryo surface.

\section{Gastrulation: Cell Mapping}

Gastrulation is the first spherical surgery in embryogenesis. It is the inevitable topological transition from the sphere with field singularities to the torus with the homogeneous field (Sect. 10), a transition that means zeroing of the field. So an

Fig. 3 Topological singularities of the discrete cell field in the crustacean Holopedium embryo: a animal hemisphere; b vegetal hemisphere (after Anderson 1973)
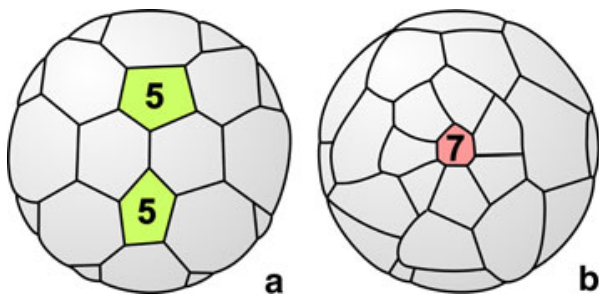
embryo or a larva is a topologically stable object and, consequently, gastrulation indeed topologically stabilizes embryonic development.

Gastrulation is also an extensive rearrangement of cells or/and epithelial sheets in an embryo. In different animals, cell streams are visualized as morphogenetic fields of cell movements - as vector fields (Fig. 4). Gilbert (2007) considered fate maps, gene expression maps and morphogenetic cell movements in developmental biology treating the mapping as a metaphor referring to the practice of cartography and as a representation of 2D surface in 3D space. However, it is possible to reveal the cell set mapping in biological morphogenesis using a topological approach. Thom (1969) was the first who considered morphogenetic cell movements topologically as a smooth mapping of the spatial patterns. Cherdantsev (2006) presented the concept of spatial unfolding of cell movements during gastrulation as the mapping of the plane into the plane. The spatial unfolding maps a flat contour into that of negative curvature. In terms of the smooth mapping theory, this means that each point of a new contour has a single prototype in the initial contour. It is possible to consider cell streams during gastrulation (Fig. 4) as the continuous mapping of the closed ball into itself (Sect. 4). In postgastrulation development, epithelial morphogenetic movements are responsible for the folding, budding, closure of separating epithelial sheets.

Topological deformations (Sect. 10) modifying connectivity of cell layers and resulting in the separation of additional closed epithelial surfaces from preexisting ones, for example, enterocoelic formation of mesoderm in Deuterostomia, neurulation, eye cap formation in chordates (Maresin and Presnov 1985; Presnov et al. 1988) can be considered as a mapping. Neural mapping is visualized due to axon connections, as, for example, retino-tectal projection via axons of retinal ganglion cells to midbrain visual centers.

\section{Morphogenesis: Spherical Surgeries}

The topological approach makes it possible to consider a succession of purely geometrical transformations of a growing organism. Our goal is a translation of anatomical descriptions into topological language, namely, to formalize the shape of an organism as a set of smooth closed surfaces. Metazoan morphogenesis may be represented as a sequence of topologically various epithelial surfaces (Isaeva et al. 2006). Epithelial cell layers are characterized by morphological and functional connectivity, closeness of an intact surface, and apical-basal polarity, i.e., outer-

Fig. 4 Morphogenetic cell movements during gastrulation: a frog embryo; b mammalian embryo (after Gilbert 2010)
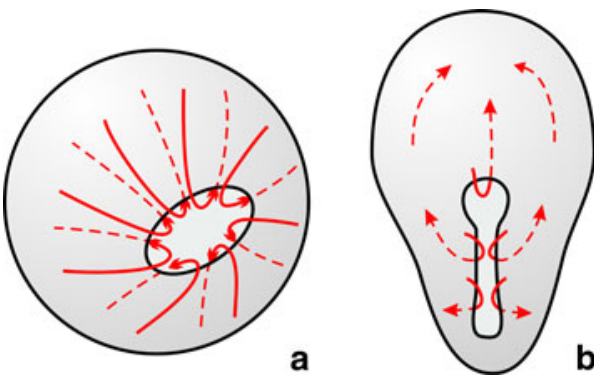
inner anisotropy. Neglecting the thickness of the cellular layer, we consider epithelia as smooth, closed, orientable surfaces, of spherical or toroidal shape. The topology of non-orientable surfaces of the Möbius strip or the Klein bottle type is impossible, both for biological membranes and for epithelial sheets.

It is natural now to apply a theorem of elementary topology to the analysis of spatial grouping of epithelial surfaces of an animal body: any closed surface in 3D space is homeomorphic to a sphere with a given number $(p)$ of handles. The sphere with $p$ handles sets a class of homeomorphic surfaces of the genus $p$. If there are no topological surgeries, such as breaking (cutting) and gluing (pasting) of epithelial sheets, the genus of the surface $(p)$ is a topological invariant, and any geometrical deformations such as surface curvature, linear and angle values are not essential. The closed surfaces of the genus $p=0$ (sphere), $p=1$ (torus), $p=2$ (double torus, or "pretzel") and so on give a topological classification.

Topological handles in biological objects are usually realized as channels (or canals, holes), for example, the digestive tube. Topological freedom in epithelial morphogenesis due to topological surgeries is realized by "cutting" and "gluing" of epithelial sheets. Those topological cases in embryogenesis occur locally, and involve complex cell events: sheet disintegration followed by cell adhesion and cytoskeletal cooperation giving rise to a newly formed cell sheet (or sheets). Furthermore, local topological surgeries inevitably lead to global topological alterations of biological forms. During embryonic development, the surface of an organism (its epithelial "envelope") undergoes sequential topological surgeries, which change the topological genus of the surface. In a case such as sea urchin development, the surface of an egg and a zygote, the outer surface of the blastula or early gastrula are surfaces of the genus 0 , whereas the epithelial surface of an embryo after gastrulation or a larva with the complete intestinal tube having both oral and anal openings is a surface of the genus 1, the torus. Accordingly, in embryonic development, the transformation of the genus of the surface, so-called spherical surgery, occurs. A transition of blind archenteron to through intestine tube is realized during gastrulation by the formation of another opening besides the blastopore (or a primary mouth); the last one becomes the definitive oral opening in Protostomia, or anal opening in Deuterostomia. This transition is the main topological profile in metazoan development.

Changes in the connectivity of germ (embryonic) layers followed by separation of additional closed spherical surfaces from preexisting ones are another kind of topological catastrophes (for example, enterocoelic formation of mesoderm, neurulation). Different types of topological surgeries modify the spatial design of epithelial sheets in ontogenesis. As a consequence, the spatial plan of an evolutionarily advanced animal organism may be represented topologically as an outer epithelial envelope of a certain genus $p$ embracing a number of inner closed epithelial surfaces embedded inside the outer envelope.

Metazoan morphogenesis may be typified as a topological pattern formation of smooth closed epithelial surfaces. Specifically, the transition from a blind archenterons to the intestine tube is the first topological step of metazoan development and evolution. In many taxa, animals have only the intestine tube without any additional channels, so their external surface is topologically equivalent 
Fig. 5 Topological body plans: a a worm; b chordate archetype (after Presnov and Isaeva 1996; Isaeva et al. 2006)
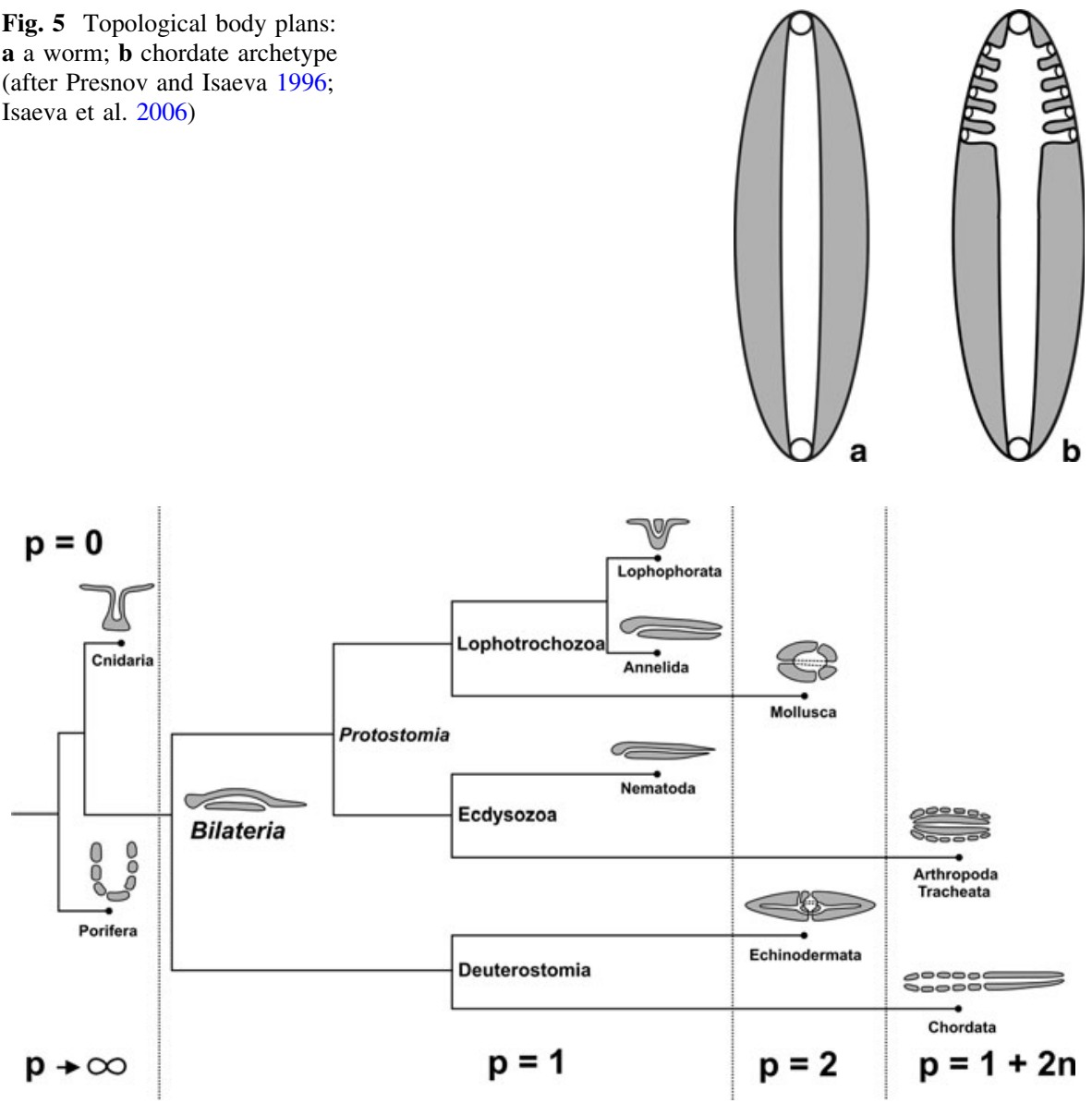

Fig. 6 Main topological transformations of animal body plans in evolution

to the torus, $p=1$ (Fig. 5a). Archetypically, the chordate body plan can be presented as the surface of genus $p=1+2 n$, which formed by an intestine and several pairs of gill slits (Fig. 5b).

Accordingly, the local topological surgery leads to the global topological complication of biological forms, as the genus of the surface is a global property. Topological dependence and topological constraints fit into evolutionary and genetically determined processes of embryogenesis-biological morphogenesis cannot be independent of the topological arrangement of physical space (schema of topological morphogenesis in Maresin and Presnov (1985)).

\section{Phylogeny: Topological Classification}

An analysis of topological image of the closed epithelial layer that covers the outer surface of an organism, regarding it as a continuous envelope formed by both 
ectodermal and endodermal (intestinal) epithelia, is applicable also to the topological evolution of the body surface in metazoan evolution. Evolutionary topological steps change the genus of the surface (Fig. 6), whereas modifications of connectivity do not change the topological scheme of the outer surface of an animal body [additional examples in Isaeva et al. (2006)]. The animal body surface is an interface between an organism and its environment. Adaptive topological transformations of the body surface in metazoan evolution result in an enlargement of the interface ensuring the optimal distribution of external medium flows within an organism, better utilization of necessary nutrients and oxygen, and more complete excretion.

\section{Conclusion: Topological Significance}

We believe that the topological approach is a powerful tool, which has not been used sufficiently in previous biological investigations. Various topological forms and functions throughout development and other topological semantics presented above bind together biological forms and functions. Local singularities and the integral morphogenetic pattern are closely linked in a developing organism and result in symmetry breaking. In other words, the topological portrait of physical space, taken as a whole, provides a sketch of fundamental consideration of biological morphogenesis, presenting a series of morphological shapes observed during development and evolution. The topological methods and theorems make it possible to consider as a whole the succession of shape transformations of metazoan organisms in development and evolution, topological correlations and restrictions, and to discover topological features and topological realization of biological morphogenesis.

Open Access This article is distributed under the terms of the Creative Commons Attribution License which permits any use, distribution, and reproduction in any medium, provided the original author(s) and the source are credited.

\section{References}

Allaerts W (1991) On the role of gravity and positional information in embryological axis formation and tissue compartmentalizaton. Acta Biotheor 39(1):47-62

Allaerts W (1999) Local and global patterns during morphogenesis of the retinotectal topographical mapping in the vertebrate brain. Acta Biotheor 47(2):99-122

Anderson DT (1973) Embryology and phylogeny in annelids and arthropods. Pergamon Press, New York

Baas NA, Seeman NC (2012) On the chemical synthesis of new topological structures. J Math Chem 50(1):220-232

Beloussov LV (1998) The dynamic architecture of a developing organism: an interdisciplinary approach to the development of organisms. Kluwer Academic Publishers, Dordrecht

Bourbaki N (1948) L'architecture des mathématiques. La mathématique ou les mathématiques? In: Les Grands Courants de la Pensée Mathématiques. Cahier du Sud, Paris, pp 35-47

Cherdantsev VG (2006) The dynamic geometry of mass cell movements in animal morphogenesis. Int J Dev Biol 50(2-3):169-182

Chuong C-M, Wu P, Plikus M, Jiang T-X, Widelitz RB (2006) Engineering stem cells into organs: topobiological transformations demonstrated by beak, feather, and other ectodermal organ morphogenesis. Curr Top Dev Biol 72:237-274

Dan-Sohkawa M, Fujisawa H (1980) Cell dynamics of the blastulation process in the starfish, Asterina pectinifera. Dev Biol 77(2):328-339 
Dubrovin BA, Novikov SP, Fomenko AT (1990, 1992, 1995) Modern geometry. Graduate texts in mathematics, vols 93, 104, 124. Springer, New York

Forman R (1998) Morse theory for cell complexes. Adv Math 134(1):90-145

Fuselier EJ, Wright GB (2009) Stability and error estimates for vector field interpolation and decomposition on the sphere with RBFs. SIAM J Numer Anal 47(5):3213-3239

Geneviere A-M, Aze A, Even Y, Imschenetzky M, Nervi C, Vitelli L (2009) Cell dynamics in early embryogenesis and pluripotent embryonic cell lines: from sea urchin to mammals. In: Rinkevich B, Matranga V (eds) Stem cells in marine organisms. Springer, Dordrecht, pp 215-244

Gibson WT, Gibson MC (2009) Cell topology, geometry, and morphogenesis in proliferating epithelia. Curr Top Dev Biol 89:87-114

Gilbert SF (2007) Fate maps, gene expression maps, and the evidentiary structure of evolutionare developmental biology. In: Laubischler MD, Maienschein J (eds) From embryology to evo-devo: a history of developmental evolution. The MIT Press, Cambridge, MA, pp 357-374

Gilbert SF (2010) Developmental biology, 9th. Sinauer Ass., Inc., Sunderland, MA

Giudice G (1962) Restitution of whole larvae from disaggregated cells of sea urchin embryos. Dev Biol 5(3):402-411

Goldstein B, Hird SN (1996) Specification of the anteroposterior axis in Caenorhabditis elegans. Development 122(5):1467-1474

Gromov M (2010) Spaces and questions. In: Alon N, Bourgain J, Connes A, Gromov M, Milman V (eds) Visions in mathematics: GAFA2000 special volume, part 1. Birkhäuser, Springer Basel AG, pp 118-161

Gromov M (2011) Crystals, proteins, stability and isoperimetry. Bull Am Math Soc 48(2):229-257

Gurwitsch AG (1922) Über den Begriff des embryonalen Feldes. Wilhelm Roux' Archiv für Entwicklungsmechanik der Organismen 52:383-415

Hinegardner RT (1975) Morphology and genetics of sea urchin development. Am Zool 15(3):679-689

Hirzebruch FEP, Kreck M (2009) On the concept of genus in topology and complex analysis. Notices Am Math Soc 56(6):713-719

Hoffman WC (1973) A system of axioms for mathematical biology. Math Biosci 16(1):11-29

Honda H (2012) Essence of shape formation of animals. Forma 27:S1-S8

Isaeva VV, Presnov EV, Chernyshev AV (2006) Topological patterns in metazoan evolution and development. Bull Math Biol 68(8):2053-2067

Isaeva VV, Kasyanov NV, Presnov EV (2008) Analysis situs of spatial-temporal architecture in biological morphogenesis. In: Kelly JT (ed) Progress in mathematical biology research. Nova Science Publishers, New York, pp 141-189

Isaeva VV, Kasyanov NV, Presnov EV (2012) Topological singularities and symmetry breaking in development. Biosystems 109(3):280-298

Jockusch H, Dress A (2003) From sphere to torus: a topological view of the metazoan body plan. Bull Math Biol 65(1):57-65

Johnson MH (2009) From mouse egg to mouse embryo: polarities, axes, and tissues. Annu Rev Cell Dev Biol 25:483-512

Johnson MH, Maro B (1985) A dissection of the mechanisms generating and stabilizing polarity in mouse 8- and 16-cell blastomeres: the role of cytoskeletal elements. J Embryol Exp Morphol 90(1):311-334

Kadokawa Y, Dan-Sohkawa M, Eguchi G (1986) Studies on mechanism of blastula formation in starfish embryos denuded of fertilization membrane. Cell Differentiation 19(2):79-88

Kirschner MW, Gerhart JC (2005) The plausibility of life. Yale University Press, New Haven

Kirschner M, Newport J, Gerhart J (1985) The timing of early developmental events in Xenopus. Trends Genetics 1:41-47

Klein DJ (2002) Topo-combinatoric categorization of quasi-local graphitic defects. Phys Chem Chem Phys 4(11):2099-2110

Knill O (2012) A discrete Gauss-Bonnet type theorem. Elem Math 67(1):1-17

Kolega J (1986) The cellular basis of epithelial morphogenesis. In: Browder W (ed) Developmental biology, vol 2. Plenum Press, New York, pp 103-143

Kyozuka K (1993) The mechanism of sperm penetration in starfish. Bull Mar Biol Station Asamushi Tôhoku Univ 19(1):1-15

Li R, Bowerman B (2010) Symmetry breaking in biology. Cold Spring Harbor Perspect Biol 2(3)a003475:1-5

Listing IB (1848) Vorstudien zur Topologie. Vandenhoeck und Ruprecht, Göttingen, pp 811-875

Maresin VM, Presnov EV (1985) Topological approach to embryogenesis. J Theor Biol 114(3):387-398 
Milnor JW (1965) Topology from the differentiable viewpoint. The University Press of Virginia, Charlottesville

Morozova N, Shubin M (2013) The geometry of morphogenesis and the morphogenetic field concept. In: Capasso V, Gromov M, Harel-Bellan A, Morozova N and Pritchard LL (eds) Pattern formation in morphogenesis: problems and mathematical issues. Springer Proceedings in Mathematics, Volume 15. Springer, Heidelberg, pp 255-282

Mullins RD (2010) Cytoskeletal mechanisms for breaking cellular symmetry. Cold Spring Harbor Perspect Biol 2(1)a003392:1-16

Newport J, Kirschner M (1982) A major developmental transition in early Xenopus embryos: II. Control of the onset of transcription. Cell 30(3):687-696

Nuccitelli R (1984) The involvement of transcellular ion currents and electric fields in pattern formation. In: Malacinski GM, Bryant SV (eds) Pattern formation: a primer in developmental biology. MacMillan, London, pp 23-46

Nüsslein-Volhard C (1991) Determination of the embryonic axes of Drosophila. Development 113(Suppl 1): $1-10$

Presnov EV (1982) Classification of biological shapes. In: Zotin AI, Presnov EV (eds) Mathematical developmental biology. Nauka, Moscow, pp 126-135 (In Russian)

Presnov EV, Malyghin SN, Isaeva VV (1988) Topological and thermodynamic structure of morphogenesis. In: Lamprecht I and Zotin AI (eds) Thermodynamics and pattern formation in biology. Walter de Gruyter, Berlin, pp 337-370

Presnov EV, Isaeva VV (1990) Positional information as symmetry of morphogenetic fields. Forma 5(1):59-61

Presnov EV, Isaeva VV (1991) Local and global aspects of biological morphogenesis. Specul Sci Technol 14(1):68-75

Presnov EV, Isaeva VV (1996) Topological classification: onto- and phylogenesis. Memorie della Societa Italiana di Scienze Naturali e del Museo Civico di Storia Naturale di Milano 27(1):89-94

Presnov EV (2008) Global decomposition of vector field on Riemannian manifolds along natural coordinates. Rep Math Phys 62(3):273-282

Presnov EV, Isaeva VV, Kasyanov NV (2010) Topological determination of early morphogenesis in Metazoa. Theory Biosci 129(4):259-270

Pyshnov MB (1980) Topological solution for cell proliferation in intestinal crypt. 1. Elastic growth without cell loss. J Theor Biol 87(1):189-200

Rashevsky N (1958) A note on biotopology of reproduction. Bull Math Biophys 20(3):275-280

Reima I, Lehtonen E (1985) Localization of nonerythroid spectrin and actin in mouse oocytes and preimplantation embryos. Differentiation 30(1): 68-75

Rossi M (2006) Natural architecture and constructed forms: structure and surfaces from idea to drawing. Nexus Netw J 8(1):112-122

Rumpler M, Woesz A, Dunlop JWC, van Dongen JT, Fratzl P (2008) The effect of geometry on threedimensional tissue growth. J R Soc Interface 5(27):1173-1180

Sawada T, Osanai K (1985) Distribution of actin filaments in fertilized egg of the ascidian Ciona intestinalis. Dev Biol 111(1):260-265

Sawada T (1988) The mechanism of ooplasmic segregation in the ascidian egg. Zool Sci 5(3):667-675

Shapiro BM, Schackmann RW, Gabel CA (1981) Molecular approaches to the study of fertilization. Annu Rev Biochem 50:815-843

Shimizu H (2012) 3D cell arrangement and its pathologic change. Forma 27:S9-S19

Shimizu T (1988) Localization of actin networks during early development of Tubifex embryos. Dev Biol 125(2):321-331

Shimizu T (1989) Asymmetric segregation and polarized redistribution of the pole plasm during early cleavages in the Tubifex embryo: role of actin networks and mitotic apparatus. Dev Growth Diff 31(3):283-298

Thom R (1969) Topological models in biology. Topology 8(3):313-335

Thom R (1996) Qualitative and quantitative in evolutionary theory with some thoughts on Aristotelian biology. Memorie della Societa Italiana di Scienze Naturali e del Museo Civico di Storia Naturale di Milano 27(1):115-117

Yamanaka Y, Ralston A, Stephenson RO, Rossant J (2006) Cell and molecular regulation of the mouse blastocyst. Dev Dyn 235(9):2301-2314

Zallen JA (2007) Planar polarity and tissue morphogenesis. Cell 129(6):1051-1063 